\title{
Extensions of Spivey's Bell number formula
}

\author{
Aimin $\mathrm{Xu}$ * \\ Institute of Mathematics \\ Zhejiang Wanli University \\ Ningbo 315100, China \\ xuaimin1009@yahoo.com.cn
}

Submitted: Dec 30, 2011; Accepted: Mar 18, 2012; Published: Apr 7, 2012

Mathematics Subject Classifications: 11B73, 11B83

\begin{abstract}
We establish an extension of Spivey's Bell number formula and its associated Bell polynomial extension by using Hsu-Shiue's generalized Stirling numbers. By means of the extension of Spivey's Bell number formula we also extend Gould-Quaintance's new Bell number formulas.
\end{abstract}

Keywords: Stirling number; Generating function; Bell number

\section{Introduction}

Stirling numbers of the second kind $S(n, k)$ have played an important role in combinatorics [7], and their sums $B_{n}$, the Bell numbers, which count the number of partitions of a set of $n$ elements, are given by

$$
B_{n}=\sum_{k=0}^{n} S(n, k), \quad n=0,1, \ldots
$$

Another well-known expression for the Bell numbers is the following

$$
B_{n}=\sum_{k=0}^{n-1}\left(\begin{array}{c}
n-1 \\
k
\end{array}\right) B_{k} .
$$

Recently, Spivey [20] discovered an interesting formula for the $(n+m)$ th Bell number:

$$
B_{n+m}=\sum_{k=0}^{n} \sum_{j=0}^{m} j^{n-k} S(m, j)\left(\begin{array}{l}
n \\
k
\end{array}\right) B_{k} .
$$

*Supported by the Zhejiang Province Natural Science Foundation (Grant No. Y6110310), and the Ningbo Natural Science Foundation (Grant No. 2010A610099). 
This new formula includes both (1) and (2) as special cases and Spivey's proof was combinatorial. Gould and Quaintance [11] provided a generating function proof of Spivey's result and used Spivey's formula to obtain a new formula for $B_{n}$. Furthermore, they extended these results to ordinary single variable Bell polynomials. Independently from Gould and Quaintance, Belbachir and Mihoubi [1] also obtained the same identity (15) in [11] and their proof follows a different approach. More recently, by using Faà di Bruno's formula $[9,12]$ for higher order derivatives of composite functions, $\mathrm{Xu}$ and Cen [22] obtained some recurrence sequences including the Bell polynomials.

In this short paper, following the technique of generating functions from Gould and Quaintance [11], we establish an extension of Spivey's Bell number formula and its Bell polynomial extension by using Hsu-Shiue's generalized Stirling numbers [14]. These results also generalize the identities for the Lah numbers discovered by Xu and Cen [22]. Moreover, we extend the new Bell number formulas due to Gould and Quaintance [11].

\section{Generalized Stirling numbers and the associated Bell numbers}

We use $(x)^{(k, \theta)}=x(x-\theta) \cdots(x-k \theta+\theta)$ to denote the generalized $k$ th falling factorial of $x$ with increment $\theta$. By starting with transformations between generalized factorial involving three arbitrary parameters $\alpha, \beta$ and $r$, Hsu and Shiue [14] introduced the generalized numbers $S(n, k ; \alpha, \beta, r)$ and unified those generalizations of the Stirling numbers due to Riordan [19], Carlitz [3, 4], Howard [13], Charalambides-Koutras [6], Gould-Hopper [10], Tsylova [21] and others. They defined a Stirling-type pair $\{S(n, k ; \alpha, \beta, r), s(n, k ; \alpha, \beta, r)\}$ by

$$
\begin{aligned}
& (x)^{(n, \alpha)}=\sum_{k=0}^{n} S(n, k ; \alpha, \beta, r)(x-r)^{(k, \beta)}, \\
& (x)^{(n, \beta)}=\sum_{k=0}^{n} s(n, k ; \alpha, \beta, r)(x+r)^{(k, \alpha)} .
\end{aligned}
$$

Especially, $S(n, k ; 0,1,0)$ is the classical Stirling number of the second kind which is denoted by $S(n, k), S(n, k ;-1,1,0)$ is the unsigned Lah number which is equal to $\frac{n !}{k !}\left(\begin{array}{l}n-1 \\ k-1\end{array}\right)$, and $S(n, k ; 0,0,1)$ is the binomial coefficient $\left(\begin{array}{l}n \\ k\end{array}\right)$. In [14], Hsu and Shiue systematically investigated many basic properties including orthogonality relations, recurrence relations, generating function and the Dobinski identity for their Stirling numbers. For more generalized Stirling numbers, one is referred to $[2,5,8,15]$.

Lemma 1 ([14]). The Stirling-type pair $\{S(n, k ; \alpha, \beta, r), s(n, k ; \alpha, \beta, r)\}$ has the following orthogonality relations

$$
\sum_{k=m}^{n} S(n, k ; \alpha, \beta, r) s(k, m ; \alpha, \beta, r)=\sum_{k=m}^{n} s(n, k ; \alpha, \beta, r) S(k, m ; \alpha, \beta, r)=\delta_{m, n},
$$

where $\delta_{m, n}$ is the Kronecker symbol. 
Lemma $2([14])$. The sequence $\{S(n, k ; \alpha, \beta, r)\}$ has the following exponential generating function

$$
\sum_{n=0}^{\infty} S(n, k ; \alpha, \beta, r) \frac{t^{n}}{n !}=\frac{1}{k !}(1+\alpha t)^{r / \alpha}\left(\frac{(1+\alpha t)^{\frac{\beta}{\alpha}}-1}{\beta}\right)^{k}
$$

The generalized Bell numbers $B_{n ; \alpha, \beta, r}$ and the polynomials $B_{n ; \alpha, \beta, r}(x)$ are defined, respectively, by the sums

$$
\begin{aligned}
& B_{n ; \alpha, \beta, r}=\sum_{k=0}^{n} S(n, k ; \alpha, \beta, r), \\
& B_{n ; \alpha, \beta, r}(x)=\sum_{k=0}^{n} S(n, k ; \alpha, \beta, r) x^{k}, \quad n=1,2, \ldots,
\end{aligned}
$$

with $B_{0 ; \alpha, \beta, r}=1$ by convention. Using (7) we easily get the generating function for the polynomials $B_{n ; \alpha, \beta, r}(x)$ and the numbers $B_{n ; \alpha, \beta, r}$.

Lemma 3 ([14]). The sequence $\left\{B_{n ; \alpha, \beta, r}(x)\right\}$ has the following exponential generating function

$$
\sum_{n=0}^{\infty} B_{n ; \alpha, \beta, r}(x) \frac{t^{n}}{n !}=(1+\alpha t)^{r / \alpha} e^{\left((1+\alpha t)^{\frac{\beta}{\alpha}}-1\right) \frac{x}{\beta}}
$$

In particular, the generating function for the generalized Bell numbers $B_{n ; \alpha, \beta, r}$ is

$$
\sum_{n=0}^{\infty} B_{n ; \alpha, \beta, r} \frac{t^{n}}{n !}=(1+\alpha t)^{r / \alpha} e^{\frac{(1+\alpha t)^{\frac{\beta}{\alpha}}-1}{\beta}}
$$

\section{Some extensions of Spivey's Bell number formula}

In this section, we will present our main results.

Theorem 4. The generalized Bell polynomials $B_{n ; \alpha, \beta, r}(x)$ satisfy the following recurrence relation

$$
B_{n+m ; \alpha, \beta, r}(x)=\sum_{k=0}^{n} \sum_{j=0}^{m}\left(\begin{array}{l}
n \\
k
\end{array}\right) x^{j} B_{k ; \alpha, \beta, r}(x) S(m, j ; \alpha, \beta, r) \prod_{i=0}^{n-k-1}(j \beta-(m+i) \alpha) .
$$

Proof. For simplicity, we denote by $\Phi(t ; x)$ the generating function for the generalized Bell polynomials on the right hand side of Eq. (10). On one hand, by the Taylor theorem we have

$$
\Phi(u+v ; x)=\sum_{m=0}^{\infty} D_{u}^{m} \Phi(u ; x) \frac{v^{m}}{m !} .
$$


Applying (10) in the above yields

$$
\Phi(u+v ; x)=\sum_{n, m=0}^{\infty} B_{n+m ; \alpha, \beta, r}(x) \frac{u^{n}}{n !} \frac{v^{m}}{m !} .
$$

On the other hand, we have

$$
\begin{aligned}
\Phi(u+v ; x) & =\Phi(u ; x)\left[\left(1+\frac{\alpha v}{1+\alpha u}\right)^{\frac{r}{\alpha}} e^{\left.\frac{x}{\beta}(1+\alpha u)^{\frac{\beta}{\alpha}}\left(\left(1+\frac{\alpha v}{1+\alpha u}\right)^{\frac{\beta}{\alpha}}-1\right)\right]}\right. \\
& =\Phi(u ; x)\left(1+\frac{\alpha v}{1+\alpha u}\right)^{\frac{r}{\alpha}} \sum_{j=0}^{\infty} \frac{1}{j !}(1+\alpha u)^{\frac{j \beta}{\alpha}} x^{j}\left(\frac{\left(1+\frac{\alpha v}{1+\alpha u}\right)^{\frac{\beta}{\alpha}}-1}{\beta}\right)^{j} .
\end{aligned}
$$

Applying (10) and (7) we obtain

$$
\begin{aligned}
\Phi(u+v ; x) & =\sum_{k=0}^{\infty} B_{k ; \alpha, \beta, r}(x) \frac{u^{k}}{k !} \sum_{j=0}^{\infty}(1+\alpha u)^{\frac{j \beta}{\alpha}} x^{j} \sum_{m=0}^{\infty} \frac{1}{m !} S(m, j ; \alpha, \beta, r)\left(\frac{v}{1+\alpha u}\right)^{m} \\
& =\sum_{k=0}^{\infty} B_{k ; \alpha, \beta, r}(x) \frac{u^{k}}{k !} \sum_{m, j=0}^{\infty} \frac{1}{m !}(1+\alpha u)^{\frac{j \beta}{\alpha}-m} x^{j} S(m, j ; \alpha, \beta, r) v^{m} \\
& =\sum_{k=0}^{\infty} B_{k ; \alpha, \beta, r}(x) \frac{u^{k}}{k !} \sum_{m, j=0}^{\infty} \frac{1}{m !} S(m, j ; \alpha, \beta, r) x^{j} v^{m} \sum_{i=0}^{\infty}\left(\begin{array}{c}
\frac{j \beta}{\alpha}-m \\
i
\end{array}\right) \alpha^{i} u^{i} .
\end{aligned}
$$

The Cauchy product rule gives

$$
\Phi(u+v ; x)=\sum_{n, m=0}^{\infty} \frac{u^{n}}{n !} \frac{v^{m}}{m !} \sum_{j=0}^{\infty} S(m, j ; \alpha, \beta, r) x^{j} \sum_{k=0}^{n}\left(\begin{array}{l}
n \\
k
\end{array}\right) B_{k ; \alpha, \beta, r}(x) \prod_{i=0}^{n-k-1}(j \beta-(m+i) \alpha) .
$$

Equating the coefficients of $\frac{u^{n}}{n !} \frac{v^{m}}{m !}$ in (13) and (14) implies that (12) is true and this completes the proof.

Example 5. Letting $\alpha=0, \beta=1$ and $r=0$ in Eq. (12) we immediately obtain

$$
B_{n+m}(x)=\sum_{k=0}^{n} \sum_{j=0}^{m}\left(\begin{array}{l}
n \\
k
\end{array}\right) x^{j} B_{k}(x) S(m, j) j^{n-k}
$$

which is a Bell polynomial extension of Eq. (3); see $[1,11]$.

Example 6. Let us denote the unsigned Lah number by $L(m, j)=\left(\begin{array}{c}m-1 \\ j-1\end{array}\right) \frac{m !}{j !}$ and $\psi_{m}(x)=$ $\sum_{i=0}^{m} L(m, i) x^{i}$. Setting $\alpha=-1, \beta=1$ and $r=0$ in (12), we rediscover the recurrence 
relation for $\psi_{m}(x)$ associated with the Lah numbers. The sequence $\left\{\psi_{m}(x)\right\}$ satisfies the following recurrence relation

$$
\psi_{n+m}(x)=\sum_{k=0}^{n} \sum_{j=0}^{m}\left(\begin{array}{l}
n \\
k
\end{array}\right) x^{j} \psi_{k}(x) L(m, j)(n-k) !\left(\begin{array}{c}
-j-m \\
n-k
\end{array}\right) .
$$

In [22], the authors gave a proof by using Faà di Bruno's formula $[9,12]$ for higher derivatives of a composite function.

Example 7. Letting $\alpha=\beta=0$ and $r=1$ in (12), we have

$$
(1+x)^{n+m}=(1+x)^{n} \sum_{j=0}^{m}\left(\begin{array}{c}
m \\
j
\end{array}\right) x^{j} .
$$

Letting $x=1$ in Eq. (12) we have the generalized Spivey's Bell number formula.

Corollary 8. The generalized Bell numbers $B_{n ; \alpha, \beta, r}$ satisfy the following recurrence relation

$$
B_{n+m ; \alpha, \beta, r}=\sum_{k=0}^{n} \sum_{j=0}^{m}\left(\begin{array}{l}
n \\
k
\end{array}\right) B_{k ; \alpha, \beta, r} S(m, j ; \alpha, \beta, r) \prod_{i=0}^{n-k-1}(j \beta-(m+i) \alpha) .
$$

It is obvious that (3) is a special case of (15) with $\alpha=0, \beta=1$ and $r=0$. It is worth noting that (3) can be extended to the $r$ Bell number [18]. In a recent paper, Mező [17] gave an $r$-Stirling extension of Spivey's formula which is a variant of (15) with $\alpha=0, \beta=1$. As another special case $r=0, \alpha=-a$ and $\beta=b$, we rediscover Theorem 5.3 given by Mansour et. al [16]. They gave a nice and attractive combinatorial proof which is very different from ours.

In the following, we are particularly interested in the case $\alpha=0$. In this case, there holds

$$
B_{n+m ; 0, \beta, r}(x)=\sum_{k=0}^{n} \sum_{j=0}^{m}\left(\begin{array}{l}
n \\
k
\end{array}\right) x^{j} B_{k ; 0, \beta, r}(x) S(m, j ; 0, \beta, r)(j \beta)^{n-k} .
$$

As a consequence, we have the following Lemma.

Lemma 9. Let $n \geqslant 0$ and $p \geqslant 0$. We have

$$
\sum_{m=0}^{p} B_{n+m ; 0, \beta, r}(x) s(p, m ; 0, \beta, r)=x^{p} \sum_{k=0}^{n}\left(\begin{array}{l}
n \\
k
\end{array}\right) B_{n-k ; 0, \beta, r}(x)(p \beta)^{k} .
$$


Proof. By Eq. (16) we can show that

$$
\begin{aligned}
& \sum_{m=0}^{p} B_{n+m ; 0, \beta, r}(x) s(p, m ; 0, \beta, r) \\
= & \sum_{k=0}^{n}\left(\begin{array}{l}
n \\
k
\end{array}\right) B_{n-k ; 0, \beta, r}(x) \beta^{k} \sum_{m=0}^{p} \sum_{j=0}^{m} x^{j} S(m, j ; 0, \beta, r) s(p, m ; 0, \beta, r) j^{k} \\
= & \sum_{k=0}^{n}\left(\begin{array}{l}
n \\
k
\end{array}\right) B_{n-k ; 0, \beta, r}(x) \beta^{k} \sum_{j=0}^{m} j^{k} x^{j} \sum_{m=j}^{p} s(p, m ; 0, \beta, r) S(m, j ; 0, \beta, r) .
\end{aligned}
$$

According to Eq. (6) with $\alpha=0$, we have

$$
\sum_{m=0}^{p} B_{n+m ; 0, \beta, r}(x) s(p, m ; 0, \beta, r)=x^{p} \sum_{k=0}^{n}\left(\begin{array}{l}
n \\
k
\end{array}\right) B_{n-k ; 0, \beta, r}(x)(p \beta)^{k},
$$

and this completes the proof.

Making use of (16) we immediately get a new identity for $B_{n ; 0, \beta, r}(x)$.

Theorem 10. Let $n \geqslant 0$ and $p \geqslant 0$. We have

$$
B_{n ; 0, \beta, r}(x)=x^{-p} \sum_{k=0}^{n}(-p \beta)^{n-k}\left(\begin{array}{l}
n \\
k
\end{array}\right) \sum_{m=0}^{p} B_{k+m ; 0, \beta, r}(x) s(p, m ; 0, \beta, r) .
$$

Proof. Replacing $k$ by $n-k$ in (17) yields

$$
(p \beta)^{-n} \sum_{m=0}^{p} B_{n+m ; 0, \beta, r}(x) s(p, m ; 0, \beta, r)=x^{p} \sum_{k=0}^{n}\left(\begin{array}{l}
n \\
k
\end{array}\right) B_{k ; 0, \beta, r}(x)(p \beta)^{-k} .
$$

Since $f(n)=\sum_{k=0}^{n}\left(\begin{array}{l}n \\ k\end{array}\right) g(k)$ is equivalent to $g(n)=\sum_{k=0}^{n}(-1)^{n-k}\left(\begin{array}{l}n \\ k\end{array}\right) f(k)$, by letting $f(n)=(p \beta)^{-n} \sum_{m=0}^{p} B_{n+m ; 0, \beta, r}(x) s(p, m ; 0, \beta, r)$ and $g(k)=B_{k ; 0, \beta, r}(x)(p \beta)^{-k}$ we have

$$
x^{p} B_{n ; 0, \beta, r}(x)(p \beta)^{-n}=\sum_{k=0}^{n}(-1)^{n-k}\left(\begin{array}{l}
n \\
k
\end{array}\right)(p \beta)^{-k} \sum_{m=0}^{p} B_{k+m ; 0, \beta, r}(x) s(p, m ; 0, \beta, r),
$$

which is equivalent to (18). This completes the proof.

It is worth noticing that, if we let $\beta=1$ and $r=0$ in (16), (17) and (18), we get the Bell polynomial extensions of Spivey's Bell number formula [11].

\section{Acknowledgements}

I would like to thank the referee for his/her useful comments and suggestions, and for drawing my attention to the articles $[17,18]$. 


\section{References}

[1] H. Belbachir, M. Mihoubi, A generalized recurrence for Bell's polynomials: An alternate approach to Spivey and Gould-Quaintance formulas, European J. Combin., 30: 1254-1256, 2009.

[2] P. Blasiak, Combinatorics of Boson normal ordering and some applictions, $\mathrm{PhD}$ Thesis, University of Paris VI and Polish Academy of Sciences, Krakow, Poland, 2005. arXiv: quant-ph/0507206.

[3] L. Carlitz, Degenerate Stirling, Bernoulli and Eulerian numbers, Utilitas Math., 15: 51-88, 1979.

[4] L. Carlitz, Weighted Stirling numbers of the first and second kind-I, II, Fibonacci Quart., 18: 242-257, 1980.

[5] L. Carlitz, M.S. Klamkin, Stirling operators, Collect.Math., XXV(2): 186-211, 1974.

[6] C. A. Charalambides and M. Koutras, On the differences of the generalized factorials at an arbitrary point and their combinatorial applications, Discrete Math., 47: 183201, 1987.

[7] L. Comtet, Advanced combinatorics, D. Reidel Publishing Co., Dordrecht, 1974.

[8] B.S. El-Desouky, N.P. Cakić, T. Mansour, Modified approach to generalized Stirling numbers via differential operators, Appl. Math. Lett., 23: 115-120, 2010.

[9] C.F. Faà di Bruno, Note sur nouvelle formule de calcul differentiel, Quarterly J. Pure. Appl. Math., 1: 359-360, 1857.

[10] H.W. Gould and A.T. Hopper, Operational formulas connected with two generalizations of Hermit polynomials, Duke Math. J., 29: 51-63, 1962.

[11] H.W. Gould, and J. Quaintance, Implications of Spivey's Bell number formula, J. Integer Seq., 11: Art. 08.3.7, 2008.

[12] W.P. Johnson, The curious history of Faà di Bruno's formula, Amer. Math. Monthly, 109: 217-234, 2002.

[13] F.T. Howard, Degenerate weighted Stirling numbers, Discrete Math., 57: 45-58, 1985.

[14] L.C. Hsu and P.J-S. Shiue, A unified approach to generalized Stirling numbers, Adv. in Appl. Math., 20: 366-384, 1998.

[15] W. Lang, On generalizations of the Stirling number triangles, J. Integer Seq., 3(2): Art. 00.2.4, 2000.

[16] T. Mansour, M. Schork and M. Shattuck, On a new family of generalized Stirling and Bell numbers, Electron. J. Comb., 18: \#P77, 2011.

[17] I. Mezö, The $r$-Bell numbers, J. Integer Seq., 14(1): Art. 11.1.1, 2011.

[18] I. Mezö, The dual of Spivey's Bell number Formula, J. Integer Seq., 15(2): Art. 12.2.4, 2012. 
[19] J. Riordan, Moment recurrence relations for binomial Poisson and hypergeometric frequency distribution, Ann. Math. Statist., 8: 103-111, 1937.

[20] M.Z. Spivey, A generalized recurrence for Bell numbers, J. Integer Seq., 11: Art. 08.2.5, 2008.

[21] E. G. Tsylova, The asymptotic behavior of generalized Stirling numbers, In Combinatorial-Algebraic Methods in Applied Mathematics, volume 158, pages 143154, 158, Gorkov. Gos. Univ., Gorkin, 1985 (in Russian).

[22] A.M. Xu, Z.D. Cen, A unified approach to some recurrence sequences via Faà di Bruno's formula, Comput. Math. Appl., 62(1): 253-260, 2011. 